\title{
Les dimensions formatives de nouvelles pratiques du conseil aux adultes: deux études de cas
}

\section{Deli Salini}

Le but de cet article est de mettre en évidence les dimensions formatives des pratiques du conseil aux adultes. À partir d'une analyse transversale de deux études de cas portant sur des pratiques récentes dans ce domaine, seront exposés quelques éléments qui participent de l'émergence de nouvelles connaissances chez les individus concernés. Ces éléments sont: i) l'accès facilité et le recours pertinent à une sélection d'informations; ii) l'accompagnement dans l'élaboration du questionnement sur les projets de développement professionnel ou de formation des adultes concernés. Ces éléments sont rendus possibles grâce à la mise en auvre par les conseillers de modalités de transmission de nouvelles connaissances, dans lesquelles les dimensions mimétiques, dialogiques et fictionnelles sont particulièrement présentes.

Introduction: Le conseil aux adultes comme situation de formation

L'activité de conseil aux adultes, initialement et principalement axée sur l'orientation scolaire et professionnelle, est devenue au cours des années une partie intégrante des stratégies d'éducation et de formation tout au long de la vie. Plusieurs études en soulignent les dimensions formatives si bien qu'elle est actuellement considérée comme une action-clé pour créer des environnements d'apprentissage ouverts, attrayants et accessibles (e.g. Cedefop, 2011; Conseil de l'Union Européenne, 2008; Guichard \& Vignoli, 2010; Jacquin \& Juhel, 2013; Kraus, 2013; Savickas et al., 2010).

Les pratiques dans ce domaine présentent une grande diversité qui s'est encore accentuée au cours de ces dernières années: on observe une extension ou transformation des formes de conseil et l'émergence de nouvelles modalités d'intervention en réponse à l'évolution des exigences du public, des prescriptions ou des contextes de référence (Mayen, 2007; Paul, 2009; Savickas et al., 2010; Zannini, 2005). Les approches qui guident l'activité des conseillers ${ }^{1}$ dans ces pratiques sont multiples et connaissent une évolution constante, caractérisée 
par le passage progressif d'un modèle de type linéaire et prédictif à un modèle constructionniste de l'orientation, à partir de perspectives qui soulignent les dynamiques non linéaires d'évolution des individus (e.g. Jacquin \& Juhel, 2013; Savickas et al., 2010).

Cette prise en compte de l'activité de conseil en tant que situation favorisant des apprentissages s'inscrit également dans le mouvement qui, depuis les années $60 \mathrm{du}$ siècle dernier, souligne l'importance des contextes non scolaires comme lieux de formation (e.g. Brougère \& Bézille, 2007; Cristol \& Muller, 2013; Maulini \& Montandon, 2005). Au sein de ce mouvement, la distinction officielle du Cedefop (2008) entre apprentissage formel, informel et non formel (Tableau 1) suscite de multiples discussions dont nous retenons deux aspects principaux. Premièrement, les trois catégories d'apprentissage indiquées ne sont pas à considérer comme parfaitement distinctes, mais comme: a) se situant sur un continuum où les éléments d'intentionnalité et de prise de conscience sont de plus en plus présents, b) allant de l'implicite à l'explicite, c) manifestant une formalisation progressive des contextes formatifs, depuis un registre du fortuit et de l'accidentel jusqu'à un registre de plus en plus institutionnalisé. Deuxièmement, il faut considérer que toute situation d'apprentissage (au-delà des distinctions proposées par le Cedefop) se caractérise par ses dimensions situées, expérientielles et participatives (Brougère \& Bézille, 2007; Cristol \& Muller, 2013).

\section{Tableau 1. Définition des trois formes d'apprentissage selon le Cedefop (2008)}

Apprentissage formel

Apprentissage dispensé dans un contexte organisé et structuré (...) et explicitement désigné comme apprentissage (...). Il débouche généralement sur la validation et la certification (p. 36).

Apprentissage informel

Apprentissage découlant des activités de la vie quotidienne liées au travail, à la famille ou aux loisirs.

Il n'est ni organisé ni structuré (en termes d'objectifs, de temps ou de ressources). L'apprentissage informel possède la plupart du temps un caractère non intentionnel de la part de l'apprenant (p. 94).

Apprentissage non formel

Apprentissage intégré dans des activités planifiées qui ne sont pas explicitement désignées comme activités d'apprentissage (en termes d'objectifs, de temps ou de ressources). L'apprentissage non formel est intentionnel de la part de l'apprenant (p. 134).

À la lumière de l'évolution des conceptions aussi bien du conseil en orientation que des contextes d'apprentissage, nous considérons les situations de conseil aux adultes comme partie intégrante du parcours d'évolution des connaissances de ceux qui y ont recours. Bien qu'elles ne soient pas caractérisées par une structure scolaire, elles offrent à leurs bénéficiaires des possibilités d'autoévaluation de leurs potentialités, lacunes et besoins, et leur procurent des occasions de transformer ou consolider leurs savoirs. En outre, en tant qu'accompagnement dans un moment de transition de vie, elles proposent une relation de service fondamentalement tournée vers l'avenir, ce qui implique aussi, pour les individus 
concernés, une transformation des significations et des connaissances relatives à eux-mêmes et à leurs activités, ainsi que des anticipations portant sur les évènements de leur vie (e.g. Mayen, 2007; Paul, 2009; Salini, 2013; Zannini, 2005).

Nous explorons la spécificité des dimensions formatives des situations de conseil aux adultes à partir d'une analyse transversale des résultats de deux études empiriques portant sur des pratiques récentes dans ce domaine. La première, réalisée en Suisse, concernait le conseil initial en validation des acquis de l'expérience (VAE) (Salini, 2013); la seconde portait sur des pratiques de conseil en évolution professionnelle (CEP) en France (Salini, 2015). Au-delà des spécificités de chaque domaine, ces deux études ont permis de relever l'importance des dynamiques de formation et d'apprentissage en jeu dans ces situations de conseil, qui découlent à la fois des modalités d'intervention des conseillers et des parcours de signification des bénéficiaires.

Après avoir présenté le cadre théorique et méthodologique de référence, nous décrivons les éléments contextuels et la procédure de recherche, ainsi que les résultats transversaux des deux études. La discussion portera ensuite principalement sur les dimensions formatives des pratiques de conseil aux adultes.

\section{Un lien indissociable entre activité, signification et apprentissage}

Nos études s'inscrivent dans une perspective d'anthropologie enactive, telle qu' élaborée par Theureau $(2006,2015)$ dans le programme de recherche empirique et technologique "Cours d'action» (CdA). Cette perspective est fondée sur trois postulats: a) le lien indissociable entre activité, cognition et signification, désigné par la notion d' «enaction" (Varela, Thompson \& Rosch, 1993); b) la dimension vécue de l'activité et la possibilité d'exprimer cette expérience, désignée par la notion de "conscience préréflexive» (Sartre, 1943); c) la dynamique permanente de transformation de l'activité humaine et de ses significations, désignée par la notion de «sémiose» et appréhendée ici en référence à la sémiotique de Peirce (Peirce, 1994; Theureau, 2006).

La notion d'enaction repose sur l'idée que la cognition se manifeste comme propriété émergeant d'une dynamique de codétermination asymétrique entre un organisme et son environnement. Elle présuppose plus précisément que: a) la cognition se caractérise par la création de significations; b) le monde n'est pas un objet externe représenté «dans» le cerveau, mais un domaine relationnel émergeant de l'interconnexion entre organisme et environnement; c) l'expérience est une dimension centrale pour la compréhension de l'activité humaine (Varela, Thompson \& Rosch, 1993).

Dans le programme CdA, la prise en compte de l'expérience se fonde sur une double hypothèse: d'une part, l'expérience correspond à une modalité de conscience particulière, c'est-à-dire une "présence à soi» ou une familiarité de 
l'acteur au flux de son activité dont le vécu est susceptible d'une compréhension que des conditions d'étude particulières permettent d'explorer (Sartre, 1943; Theureau, 2006). D'autre part, l'expérience se manifeste comme une activité interprétative continue de l'existant à partir d'une dynamique de signification, qui est conçue comme incarnée et située au-delà d'une vision exclusivement intralinguistique (Theureau, 2006). Cette dynamique se fonde sur trois registres de l'expérience interdépendants et concomitants (Peirce, 1994). Le premier est de l'ordre du Possible, il exprime les significations qui pourraient s'actualiser ou non dans une situation donnée; le deuxième est de l'ordre de l'Actuel, de ce qui est identifié par un individu comme significatif à un moment donné et dans une situation spécifique; le troisième est de l'ordre du Virtuel, de la mise en relation et de la médiation entre les catégories précédentes et il exprime la tendance à généraliser et à identifier une régularité dans les faits, ce qui permet d'en anticiper la réalisation. Ce registre correspond au «sens de l'apprentissage» car il permet de valider, infirmer ou modifier les significations constituées préalablement à une situation donnée. Dans cette perspective, les actions de signifier, apprendre ou comprendre reviennent à anticiper à partir de régularités constituées, ce qui donne lieu à des scénarios potentiels d'avenir qui pourront s'actualiser si certaines conditions sont présentes (Peirce, 1994; Theureau, 2006).

À partir des trois registres définis par Peirce (1994), on peut considérer que dans toute situation expérimentée par un individu, se manifestent des dynamiques de transformation aussi bien que de signification et d'apprentissage. Elles sont l'expression de cycles de recherche qui, soit consolident et précisent des généralisations antérieures, soit les déconstruisent avant une reconfiguration. Ce mouvement de déconstruction et reconfiguration, souvent déclenché par un élément inhabituel qui invalide les généralisations antérieures, émerge au cours d'un mouvement interprétatif (ou d'investigation). Celui-ci se caractérise initialement par des sentiments d'incertitude et d'indétermination, des recombinaisons de fragments d'anciennes connaissances, des distinctions par la négative ("ce n'est pas»), des questionnements, des successions d'hypothèses, des dialogues internes ou externalisés sur le positionnement ou la dénomination de ce qui apparaît comme inhabituel, jusqu'à l'établissement de nouvelles modalités de signification et d'une nouvelle organisation des connaissances (Peirce, 1878-1879).

Dans ce parcours interprétatif, deux composantes de la dynamique de la signification jouent un rôle essentiel: l'activité imaginative et la dimension dialogique de la pensée (Peirce, 1994). L'activité imaginative selon Peirce s'exprime par l'émergence des icônes, c'est-à-dire des images, diagrammes et métaphores. Ce sont des signes qui renvoient à leur objet par ressemblance, en s'appuyant sur tout élément sensoriel. Inscrites dans le registre d'expérience du Possible, les icônes ont une dimension fluide ou instable, qui se caractérise par du flou et de l'ouverture: d'une part elles n'ont pas de contours définis, d'autre part elles constituent des hypothèses de compréhension ou d'interprétation du monde en s'inscrivant ainsi dans un mouvement d'investigation (Fisette, 2009). Les 
relations de ressemblance par lesquelles elles s'expriment ne sont pas de l'ordre de l'objectif, elles se constituent par une mise en lien de domaines différents à partir de l'expérience individuelle, pouvant même faire référence à des objets qui n'existent pas, à l'instar des personnages fantastiques. En ce sens, elles sont l'expression d'une création (Peirce, 1994).

L'image se caractérise par une «identité partagée» entre l'individu et son objet. Elle permet de reconnaître le monde comme familier et contribue à l'émergence d'une "consonance intentionnelle» avec d'autres individus. En ce sens, les phénomènes mimétiques s'inscrivent dans une dimension de partage (Gallese, 2007). Le diagramme renvoie à son objet par une perception de similarité de ses relations, tels que les schèmes mais aussi les récits ou les pas d'une danse (Fisette, 2009). La métaphore permet de constituer des relations de ressemblance par la création de similarités inhabituelles entre des signes. Elle fait apparaittre des propriétés inédites de ce qui est mis en relation, en ouvrant des perspectives nouvelles de compréhension. Inscrite dans les dimensions sensorimotrices, affectives, sociales et culturelles de l'expérience (Lakoff \& Johnson, 1980), en tant que "fiction en miniature», elle permet de configurer des scénarios d'avenir, car elle préfigure et contient en soi les pistes de son développement possible (Genette, 2004).

En reprenant et réinterprétant les conceptions de Platon, Peirce énonce que la pensée n'est pas un monologue intérieur, mais s'inscrit dans l'altérité, dans une "conversation» entre différents états de l'esprit ou de la conscience: entre un moi présent et un moi futur, en tant que conciliabule interne et fictionnel avec des interlocuteurs imaginaires, en tant que dialogue entre les actes de pensée et les actions concrètes, ou entre les doutes, hypothèses et convictions qui traversent un mouvement d'investigation. Comme le souligne Rosenthal (2012), c'est par l'émergence d'une voix de l'intérieur que chacun est dans un rapport dialogique avec lui-même. L'externalisation de cette voix et du dialogue interne permet le développement des questions, doutes, hypothèses ou convictions qui traversent l'individu lors de son investigation. Cette dimension dialogique de la pensée participe à l'institution de la vie psychique, sociale et culturelle à la fois de l'individu et du collectif, de même qu'aux dynamiques de signification.

\section{Identifier les composantes de l'expérience vécue}

A partir de ce cadre d'analyse sémiologique de l'activité, la méthode de constitution et de traitement des données se fonde sur: a) une démarche ethnographique; b) un positionnement éthique et méthodologique garantissant une relation à l'autre non objectivante et une fiabilité des données récoltées; c) le recours à une méthode facilitant l'accès à la "conscience préréflexive» des acteurs et à l'analyse de leurs catégories d'expérience (Céfaï, 2003; Theureau, 2006). 
La prise en compte de la "conscience préréflexive» de l'acteur se fait en considérant ce qui, dans son activité, est significatif, montrable, racontable, mimable et commentable par lui. L'expression de ces éléments est facilitée par des séances de «remise en situation dynamique» (RSD) par auto-confrontation de l'acteur aux traces (souvent des enregistrements vidéo) de sa propre activité. Dans ces séances (enregistrées elles aussi), les acteurs sont invités à «revivre» les situations enregistrées et sont incités à en décrire les éléments significatifs pour eux et à les commenter, ceci grâce au support de traces qui facilitent l'accès contextualisé à l'activité vécue (Gallese, 2007; Theureau, 2006), ainsi que par des relances du chercheur permettant à l'acteur de rester au plus proche du vécu de l'activité étudiée.

L'identification du contenu des catégories de l'expérience se fait initialement par des protocoles de mise en correspondance des transcriptions de l'activité (comportement verbal et non verbal) en situation naturelle, avec la transcription de ce qui a été exprimé par les acteurs lors des séances de RSD (Tableau 2).

\section{Tableau 2. Exemple d'un protocole de mise en correspondance}

\begin{tabular}{|l|l|l|}
\hline $\mathrm{t}$ & Séance de conseil entre CoC et AoSo & RSD AoSo \\
\hline 0:02:06 & $\begin{array}{l}\text { CoC: ... toutes les branches, se résument } \\
\text { sur cette feuille. Alors ça c'est le profil de } \\
\text { qualification (appuie la feuille sur la table } \\
\text { vers AoSo, montre avec le doigt les différents } \\
\text { éléments) (...) }\end{array}$ & $\begin{array}{l}\text { AoSo: (observe attentivement la fenille) } \\
\text { Cher: Que regardez-vous là? Qu'est-ce qui } \\
\text { attire votre attention? } \\
\text { AoSo: Je regarde ça, je regarde vraiment } \\
\text { la feuille, ce qui est marqué, ce qu'ils } \\
\text { demandent, ce qu'il faut faire. C'est } \\
\text { vraiment la feuille que je regarde. J'entends } \\
\text { quand même ce qu'elle me dit, mais c'est la } \\
\text { feuille, parce que tout d'un coup, c'est ça } \\
\text { que je vais devoir savoir-faire. (...) Donc } \\
\text { là, je me dis qu'il y a des trucs que je ne } \\
\text { sais pas... }\end{array}$ \\
\hline
\end{tabular}

Note. Extrait du protocole de transcription et de mise en correspondance de la séance de conseil entre le conseiller CoC et le candidat AoSo, et de la séance de RSD avec AoSo et la chercheure Cher.

L'analyse des données ainsi constituées se fait à partir d'une matrice à six composantes (Tableau 3) qui renvoient aux trois catégories d'expérience définies par Peirce. 
Tableau 3. Matrice à six composantes pour l'analyse sémiologique de l'expérience des acteurs

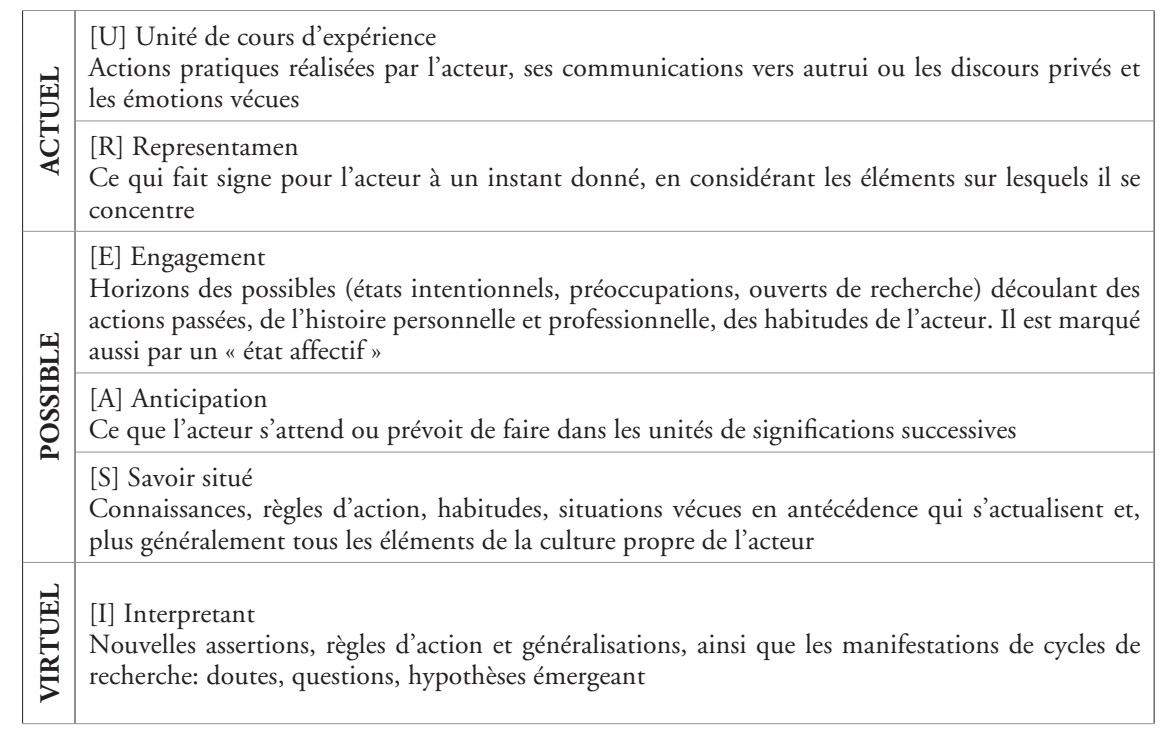

À cette démarche analytique ou de déconstruction fait suite une phase de généralisation ou de reconstruction, dans laquelle les dimensions typiques de l'expérience de chaque acteur sont répertoriées selon une classification thématique, visant à identifier les éléments principaux de la dynamique de signification des acteurs.

\section{Deux études sur des pratiques récentes de conseil}

Les deux contextes d'étude pris en compte, même s'ils reposent sur des pratiques différentes du conseil aux adultes, ont en commun les éléments suivants: a) ils s'adressent à des adultes qui s'interrogent sur leur avenir professionnel ou formatif, et sont donc impliqués explicitement dans un problème d'anticipation, b) ce sont des entretiens initiaux, c) ils visent à fournir aux bénéficiaires des informations pertinentes et adaptées à leur demande. De même, tout en poursuivant des objectifs spécifiques, les deux études a) se sont fondées sur le même cadre théorique et méthodologique; b) visaient à identifier les aspects typiques des dynamiques de signification des acteurs concernés; c) cherchaient à dégager des indications utiles pour la conception d'offres et de formation dans le domaine du conseil. L'ensemble de ces caractéristiques indique la pertinence d'une analyse transversale des données des deux études. 
La première étude (E1) a été réalisée pendant trois ans dans un service suisse de VAE et portait sur les entretiens d'information-conseil initial (SICI) (Salini, 2013). Cette forme de conseil suppose que le conseiller sache informer et conseiller les bénéficiaires sur cette modalité de qualification en devenir, afin de faciliter leur choix ou leur engagement dans la procédure. L'étude comportait 37 SICI, gérées individuellement par les quatre conseillers du service. Onze entretiens ont été retenus pour les séances de RSD, effectuées séparément pour chaque bénéficiaire et conseiller impliqué.

La seconde étude (E2) concernait le Conseil en évolution professionnelle (CEP), proposé par le Centre Régional de Ressources de Bourgogne (C2R $)^{2}$. Il s'agit d'un dispositif gratuit et personnalisé, introduit en France récemment (République française, 2014) et qui a fait l'objet de différentes expérimentations, dont celle en Bourgogne. Il est adressé à toute personne souhaitant faire le point sur sa situation professionnelle et, le cas échéant, établir un projet d'évolution professionnelle ou de formation. Nous avons rejoint ce projet à l'automne 2014 et réalisé une étude exploratoire d'une portée plus réduite que la précédente. Les données ont été collectées par l'observation, l'audio-enregistrement et la transcription de dix séances initiales de CEP et des séances subséquentes de remise en situation dynamique (RSD) (aussi audio-enregistrées) avec les trois conseillers impliqués. La récolte des données a été complétée par la prise en compte de 13 textes produits par les conseillers du C2R participant à un groupe d'analyse de pratiques (Mayen, 2015; Salini, 2015).

Pour les deux études, le traitement des données a été réalisé par un travail de déconstruction et de reconstruction du flux de l'activité des acteurs (conseillers et bénéficiaires pour l'E1, conseillers seulement pour l'E2) selon le cadre d'analyse sémiologique. L'analyse a permis d'identifier les modes de signification et les types d'intervention des conseillers et, pour l'E1, d'identifier en détail les dynamiques de signification des bénéficiaires (voir exemple dans le Tableau 4).

\section{Tableau 4. Extrait du protocole d'analyse des données de TeJo}

\begin{tabular}{|c|c|c|c|c|c|c|c|}
\hline \multirow{2}{*}{$\begin{array}{c}\text { Séance TeJo } \\
\text { et } \mathrm{CoB}\end{array}$} & \multirow[t]{2}{*}{ RSD TeJo } & \multicolumn{6}{|c|}{ Analyse en six composantes } \\
\hline & & {$[\mathrm{R}]$} & {$[\mathrm{U}]$} & {$[\mathrm{E}]$} & {$[\mathrm{A}]$} & {$[S]$} & {$[\mathrm{I}]$} \\
\hline $\begin{array}{l}\text { CoB : D'accord. } \\
\text { Si vous voulez, } \\
\text { nous ce qu'on } \\
\text { attend de } \\
\text { vous, c'est que } \\
\text { vraiment vous } \\
\text { puissiez détailler } \\
\text { ce qui vous est } \\
\text { arrivé dans un } \\
\text { moment parti- } \\
\text { culier }\end{array}$ & $\begin{array}{l}\text { TeJo : Voilà, là, } \\
\text { je commence à } \\
\text { comprendre où } \\
\text { il veut en venir. } \\
\text { Pour moi, ce } \\
\text { serait beaucoup } \\
\text { plus clair si } \\
\text { lui, il avait } \\
\text { commencé à } \\
\text { introduire qu'il } \\
\text { faut expliquer } \\
\text { plus dans les } \\
\text { détails }\end{array}$ & $\begin{array}{l}\text { L'expli- } \\
\text { cation de } \\
\text { CoB sur } \\
\text { les raisons } \\
\text { de rentrer } \\
\text { dans les } \\
\text { détails }\end{array}$ & $\begin{array}{l}\text { Impression } \\
\text { de comm- } \\
\text { encer à } \\
\text { comprendre } \\
\text { comment il } \\
\text { faut décrire } \\
\text { son activité }\end{array}$ & $\begin{array}{l}\text { Com- } \\
\text { prendre } \\
\text { comment } \\
\text { rédiger le } \\
\text { rapport de } \\
\text { preuve }\end{array}$ & $\begin{array}{l}\text { Rédiger } \\
\text { son } \\
\text { rapport } \\
\text { de preuve } \\
\text { avec } \\
\text { beaucoup } \\
\text { de détails } \\
\text { sur son } \\
\text { activité }\end{array}$ & $\begin{array}{l}\text { Il faut } \\
\text { expliquer } \\
\text { aux gens } \\
\text { ce qu'on } \\
\text { s'attend } \\
\text { d'eux }\end{array}$ & $\begin{array}{l}\text { Dans le } \\
\text { rapport de } \\
\text { preuves il } \\
\text { faut décrire } \\
\text { son activité } \\
\text { profession- } \\
\text { nelle dans } \\
\text { les détails }\end{array}$ \\
\hline
\end{tabular}


Cette analyse transversale prend donc en compte les données des deux études en mettant l'accent sur ce qui relève de l'activité des conseillers, tout en intégrant les éléments pertinents concernant l'activité des bénéficiaires.

Puisqu'elle se limite aux spécificités des deux contextes de conseil, elle ouvre des pistes d'interprétation qui devraient être confirmées par d'autres recherches plus étendues sur les diverses formes de conseil et sur leurs dimensions formatives.

\section{Un espace de rencontre qui participe de l'évolution du questionnement des bénéficiaires}

Les éléments transversaux des résultats de ces deux études (Salini, 2013, 2015) sont présentés ici de manière synthétique afin de faire apparaitre quelques éléments typiques de l'activité des bénéficiaires, ainsi que les caractéristiques principales de l'intervention des conseillers. Nous ne rapporterons donc pas en détail toutes les composantes de la phase de déconstruction de l'activité selon les six catégories d'analyse, mais seulement les éléments de reconstruction qui témoignent des dimensions typiques de l'activité des acteurs concernés. Nous présenterons notamment les résultats principaux qui touchent d'abord à la spécificité du mouvement explicite d'investigation des bénéficiaires et ensuite aux formes d'intervention des conseillers. Parmi ces dernières, deux formes apparaissent essentielles et interdépendantes: a) chercher des informations pertinentes et les rendre saisissables; b) accompagner l'élaboration du questionnement des bénéficiaires grâce à la mise en ouvre d'un ensemble de modalités de transmission de nouvelles connaissances, parmi lesquelles les dimensions mimétiques, dialogiques et fictionnelles sont particulièrement présentes et efficaces.

\section{Des adultes impliqués dans un mouvement \\ d'investigation explicite}

La forme d'engagement principale des bénéficiaires est celle de la quête personnelle: il s'agit d'hommes et de femmes qui s'interrogent sur leur parcours, examinent leur situation et en anticipent les évolutions, en s'adressant à des experts pour constituer une alliance portant sur leurs intentions et questionnements. Au niveau du parcours interprétatif, les propos de ces adultes manifestent leur positionnement à différentes étapes du mouvement d'investigation indiqué par Peirce (1878-1879). Certains arrivent au conseil à la suite de l'évolution imprévue de leur environnement de travail, comme $\mathrm{CeRo}^{3}$ qui, face aux changements de son métier, constate: "Je n'ai pas de vision du futur dans ce domaine...", ou FaDo, un homme qui, face aux exigences contradictoires de ses multiples interlocuteurs, doute qu'il soit un professionnel compétent et digne de confiance. Pour d'autres, ces recherches ont émergé au fil des années, nourries par le désir de trouver de nouvelles options d'évolution: «Ça fait un petit moment que ça me 
taraudait...» (KeMo). D'autres entrevoient l'arrivée de bouleversements dans leur lieu de travail et, comme AnPe, se préparent: "Je vois le résultat de la vente, la réduction des commandes...". Parfois, l'objectif est vague et les propos formulés sous forme négative: "Je ne veux plus» et en même temps "Je ne sais pas bien vers où allern, disent plusieurs personnes.

Face à ce questionnement, le moment du conseil constitue un espace de rencontre qui offre la possibilité de configurer ou de faire évoluer le projet d'avenir des bénéficiaires. Cette évolution est facilitée par la multiplicité des formes d'intervention proposées par les conseillers.

\section{Chercher et rendre saisissables les informations pertinentes}

Afin de proposer au public des renseignements fiables et actualisés et d'offrir un éventail d'options plus étendues, les conseillers doivent repérer des informations sur l'actualité législative et financière, la situation du marché du travail et des professions, les filières de formation et leur accessibilité, ainsi que sur le fonctionnement du réseau des organisations partenaires. Ce travail n'est pas réalisé seulement de manière générale, mais par la recherche explicite de détails concrets des situations. Ce travail «avec et sur les informations» (Mayen, 2007, 2015) est réalisé d'une part en l'absence des bénéficiaires. Il permet alors de sélectionner et d'organiser l'ensemble de l'information éparpillée dans les multiples contextes de référence. Il est réalisé d'autre part en situation d'entretien, pour que l'information soit entendue, comprise et intégrée. Comme le signale le $\mathrm{CoF}$, lors d'une séance de RSD: «Souvent les personnes n'ont pas les moyens de savoir tout ce qui est possible dans un domaine de formation, car c'est beaucoup compliqué, on n'a pas les clés d'entrée...». Pour ce faire, il faut des opérations de transformation qui rendent les informations accessibles et donnent lieu à des opérations d'étayage qui permettent aux bénéficiaires de s'approprier l'information, de l'intégrer et de s'en servir.

\section{Accompagner L'élaboration du questionnement de bénéficiaires}

Au cours des séances de conseil, les bénéficiaires présentent progressivement les multiples éléments auxquels ils doivent faire face ainsi que leur questionnement. Les conseillers-s'activent pour accompagner cette élaboration selon trois dynamiques principales: a) comprendre pour faire comprendre, b) participer à l'évolution du mouvement d'investigation, c) proposer des scénarios d'avenir.

- Comprendre pour faire comprendre: le pôle mimétique. Tout au long de l'entretien, les conseillers s'engagent dans un travail progressif de rapprochement à l'autre sur le mode de la résonance. Il s'agit d'un engagement intentionnel, visant à saisir les intentions, déceptions ou espoirs des bénéficiaires. Cette «consonance intentionnelle» (Gallese, 2007), qui ressortit au mimétisme, s'exprime comme une forme directe de compréhension expérientielle d'autrui. Ceci ne signifie pas 
que le vécu des autres soit ressenti comme sien: il s'agit d'un "comme si», qui peut amener la personne à ressentir le vécu d'autrui tout en s'en différenciant. Ceci est saisi par les bénéficiaires: HuMo est satisfaite de découvrir que le CoD peut la comprendre "Je me suis dit "oui il voit"). Elle s'amuse aussi en découvrant que le conseiller a bien compris l'ensemble de son activité: "Ça me fait rire parce qu'il a compris mon système, ah oui...». Ainsi que l'a souligné le $\mathrm{CoE}$, cette capacité de résonance donne aux bénéficiaires la possibilité d'accueillir les points de vue ou suggestions du conseiller, puisque celles-ci "correspondent» à leurs propres exigences, au point que, vers la fin de l'entretien, $\mathrm{FaDo}$ dit à la conseillère: «Vous arrivez même à lire dans mes pensées, je crois». Cette capacité de résonance au monde et au vécu de l'autre permet également de mieux gérer l'équilibre entre «faire à sa place» et "encourager à faire par soi-même», par l'identification du degré de soutien nécessaire.

- Participer à l'évolution du mouvement d'investigation: le pôle dialogique. La participation dialogique à l'expression et à l'évolution des doutes, questionnements, hypothèses et convictions qui caractérisent le mouvement d'investigation des bénéficiaires constitue une autre forme d'accompagnement. Au cours des entretiens, les bénéficiaires présentent peu à peu les multiples éléments de ce mouvement, par rapport auxquels les conseillers s'impliquent de différentes manières. D'une part ils y font écho, de l'autre ils y contribuent en formulant à leur tour des hypothèses ou en proposant des reformulations qui donnent une épaisseur argumentative aux propos des bénéficiaires. Par exemple, le CoE fait écho à $\mathrm{FaDo}$ par des marques d'assentiment ou de petites reformulations qui communiquent sa compréhension, facilitent l'expression et légitiment le vécu de l'autre, tout en accompagnant le développement d'un discours plus argumenté, comme dans l'extrait suivant:

FaDo (c'est) Le contact client qui me...

$\mathrm{CoE}$ oui, qui vous pèse, ... peut-être...

FaDo qui me pèse...

$\mathrm{CoE}$ ouais

FaDo qui me pèse, qui me pèse parce que... (Il évoque la colère des clients...)

CoE oui, oui. C'est... c'est compliqué parce que vous savez que pour eux c'est difficile...

FaDo voilà, c'est difficile. (...). Faut énormément prendre sur soi.

$\mathrm{CoE}$ (expression d'assentiment)

FaDo voilà, donc, ....... (souffle), faut garder son calme, faut encaisser. Et jour après jour, ça va faire (plusieurs années) que j’fais ça...

CoE: ouais

(...)

CoE ouais, ça je comprends, oui. C'est-à-dire, on vous demande de faire plusieurs choses à la fois...

FaDo oui.... 
CoE et en plus, ... dans des situations où il faut être présent pour le client, pour qu'il se sente pris au sérieux

$\mathrm{FaDo}$ tout à fait.

Dans le conseil en VAE, le questionnement des bénéficiaires concerne aussi la manière de réussir la procédure. DiRu, attend «des suggestions qui puissent m'aider à choisir la meilleure option" car il hésite entre deux secteurs possibles de validation de son activité professionnelle. De plus, dans le conseil en VAE, il arrive souvent que les propos des conseillers fassent découvrir des éléments inattendus sur la procédure, ouvrant alors pour les bénéficiaires de nouveaux parcours de recherche (Salini, 2013). TeJo par exemple est surprise qu'il ne s'agisse pas seulement de cours et n'arrive pas à comprendre ce que lui demande le conseiller: "Je ne sais pas où il voulait venir. Je ne comprenais pas la logique de ces questions", affirme-t-elle, en référence au moment où $\mathrm{CoB}$ essaie d'explorer les caractéristiques de son travail. Grâce au dialogue avec le conseiller, se développe pour elle la compréhension de la spécificité de la procédure et, vers la fin de l'entretien, elle intègre de nouvelles connaissances, notamment sur la manière de rédiger son dossier de VAE: "Il faut raconter son activité en détail», dit-elle. Cette dimension dialogique des entretiens de conseil est soulignée par Guichard (2013) qui, faisant aussi référence aux travaux de Peirce (1994) et de Jacques (1982), indique que la dimension dialogique est une des formes essentielles de l'accompagnement en orientation, parce qu'elle aide les personnes à développer leur réflexivité et à donner sens à leur existence.

- Proposer des scénarios d'avenir: le pôle fictionnel. Le travail de facilitation à l'anticipation des actions et choix futurs se réalise par le recours important à des dimensions fictionnelles, comme des métaphores, des dialogues fictifs, ainsi que par la proposition de scénarios de situations possibles. Pour illustrer une action à entreprendre, la métaphore du voyage est presque omniprésente: plusieurs conseillers proposent un schéma qui se présente comme un plan, sur lequel sont placés les éléments du parcours à envisager, les instances concernées et leurs relations, mais aussi l'attitude de «marcheur» qu'il faut garder:

CoF donc le plus (important)..., c'est de garder la motivation, d'avoir en tête toutes les étapes que vous devez passer, avec la stratégie des petits pas. Car le changement d'un métier c'est quand même un grand truc!

CeRo oui, oui!

$\mathrm{CoF}$ donc il ne faut pas que vous visiez (seulement) le sommet, il faut voir étape par étape.

D'autres métaphores peuvent aussi émerger, comme celle de la balance, pour évoquer la recherche de correspondance entre l'expérience et le titre visé par la VAE, ou celle du cœur pour évoquer un point central de la démarche. Ces formes d'explications, récurrentes dans toute pratique de conseil (Hagen \& Jordan, 2012), sont souvent associées à la production de dialogues hypothétiques dans 
lesquels on se met à la place d'autres interlocuteurs potentiels, par exemple, celle des experts qui évalueront le dossier de VAE: "Peut-être qu'ils vous disent: "ah oui, là je vois, là elle décrit cette activité-là, là, après, y en a une autre, et y en a encore une autre, je peux... vu que, oui, je vois que..." (CoC).

De même, il s'agit d'amener les bénéficiaires à se représenter divers horizons dans leur avenir, par la co-élaboration de scénarios mettant en scène ce qu'ils pourront faire ou rencontrer. Cette démarche se fonde sur l'accueil des perspectives proposées par les bénéficiaires tout en s'efforçant de les élargir, de les préciser et, notamment dans le CEP, de les diversifier. Elle permet ainsi la concrétisation et la définition des trajectoires possibles, afin de faciliter chez les bénéficiaires le passage d'une préfiguration générique et parfois inadaptée à une préfiguration située et mieux ciblée. Cet effort de concrétisation a pour conséquence que la description des contextes liés aux divers projets possibles prend corps avec des détails concrets: caractéristiques des lieux et personnes à rencontrer, stratégies pour soumettre ses exigences, coûts ou formes de financement, horaires et temps de déplacement. Le CoF indique par exemple à son interlocutrice: "Vous voyez, pour aller dans cette institution vous devez arriver en ville de Dijon, à la gare vous trouvez un tram rose, qui vous amène après quatre arrêts...». Ainsi, comme dans une pièce de théâtre, apparaissent, par simulation, les éléments scénographiques d'une situation ou d'un contexte facilitant, par mimétisme, une immersion dans un avenir possible.

\section{Pratiques de conseil et apprentissage tout au long de la vie}

Le conseil en VAE et le CEP font partie des nouvelles pratiques d'accompagnement des adultes tout au long de la vie. Sans reprendre ici les conditions de leur émergence (voir à ce propos Mayen, 2007, 2015; Salini, 2013), leur présence même souligne la conviction, de plus en plus répandue, que les adultes ne sont pas figés dans un statut stable, mais s'inscrivent dans un développement mouvant, qui les amène à profiter de tous les contextes de la vie pour étendre leurs connaissances et faire avancer leurs projets. De leur côté, les conseillers et conseillères interpellés par ces adultes combinent plusieurs formes d'intervention, qui s'expriment aussi par des stratégies éducatives non formalisées (Roquet, 2009).

Ces stratégies, qui se situent à la frontière des pratiques classiques de la formation, tiennent compte des mutations importantes du monde du travail et des nouvelles exigences qu'il suscite (Hirschi, 2012; Schiersmann et al., 2012). Citons par exemple la prolifération des rapports de travail instables, la diffusion des carrières "patchwork", l'émergence de professions nouvelles ainsi que la tendance des femmes et des hommes à vouloir concilier davantage leur vie familiale et sociale avec leur vie professionnelle. En outre, la croissance 
exponentielle des informations qui dépassent les possibilités des individus de les gérer et de s'y référer pour prendre des décisions, risque de l'emporter sur la possibilité de trouver le bon scénario à développer. En effet, ce foisonnement d'exigences et d'informations requiert de la personne non seulement qu'elle actualise continuellement ses connaissances, mais aussi qu'elle prenne ses responsabilités pour préciser, entretenir et en même temps adapter régulièrement ses projets. Ceci peut s'effectuer en présence de conseillers sachant accompagner régulièrement cette quête et concevant l'adulte comme un sujet agissant et évoluant dans une société complexe.

Les trois pôles d'intervention que nous avons relevés: mimétique, dialogique et fictionnel, nous semblent pertinents dans l'accompagnement d'individus en transition et donc, par définition, dans une situation à la fois d'incertitude et d'ouverture. La dimension mimétique offre un espace de reconnaissance de soi-même et de ses exigences, dans le respect de la complexité de toute histoire individuelle. La dimension dialogique ouvre à la prise en compte d'autres points de vue et d'autres manières de dire et de se dire, tout en légitimant ses propres convictions. Enfin, la dimension fictionnelle, rendue possible notamment par l'expertise de professionnels sachant faire face au foisonnement des informations contextuelles, ouvre à des possibles qu'on peut explorer grâce à l'alliance faite avec une personne qui, par sa présence même, fait office de miroir au projet d'avenir de ces adultes.

Ces pôles d'intervention, qui sollicitent l'évocation expérientielle et l'imaginaire à partir de propositions de compréhension basées sur la ressemblance, nous semblent particulièrement représentatifs des dimensions formatives présentes dans les pratiques de conseil. Ces dimensions relèvent de l'articulation entre un questionnement des bénéficiaires sur des choix à faire dans leur vie et l'engagement actif des conseillers qui visent l'évolution des connaissances et ainsi l'élargissement des possibles des individus qui les interpellent.

\section{Notes}

1 L'utilisation du genre masculin a été adoptée afin de faciliter la lecture et n'a aucune intention discriminatoire.

2 Cette recherche a été possible grâce à un financement du Fond national suisse et par la collaboration de l'Institut Agrosup de Dijon, que nous tenons ici à remercier.

3 Les acteurs impliqués dans les deux études sont indiqués de la manière suivante: les conseillers de l'E1 avec les codes $\mathrm{CoA} / \mathrm{CoB} / \mathrm{CoC} / \mathrm{CoD}$ et ceux de l'E2 avec le codes $\mathrm{CoE}$, $\mathrm{CoF}, \mathrm{CoG}$, tandis que les bénéficiaires sont indiqués par les initiales du prénom et du nom. 


\section{Références}

Brougère, G. \& Bézille, H. (2007). De l'usage de la notion d'informel dans le champ de l'éducation. Revue française de pédagogie, 158, 117-160.

Cedefop. (2008). Terminology of European education and training policy. A selection of 100 key terms. Luxembourg: Office for Official Publications of the European Communities.

Cedefop. (2011). Lifelong guidance across Europe: Reviewing policy progress and future prospects (Working Paper No 11). Luxembourg: Publications Office of the European Union.

Céfaï, D. (Éd.). (2003). L'enquête de terrain. Paris: La Découverte.

Conseil de l'Union Européenne (21 novembre 2008). Résolution du Conseil sur «Mieux inclure l'orientation tout au long de la vie dans les stratégies d'éducation et de formation tout au long de la vie». Journal officiel de l'UE (C 319/4 du 13.12.2008).

Cristol, D. \& Muller, A. (2013). Les apprentissages informels dans la formation pour adultes. Savoirs, 2, (32), 11-59.

Fisette, J. (2009). L'icône, l'hypoicône et la métaphore. Visual culture, 14, 7-46.

Gallese, V. (2007). Dai neuroni specchio alla consonanza intenzionale. Meccanismi neurofisiologici dell'intersoggettività. Rivista di psicoanalisi, LIII, (1), 197-208.

Genette, G. (2004). Métalepse. De la figure à la fiction. Paris: Le Seuil.

Guichard, J. (2013). Quel paradigme pour des interventions d'accompagnement en orientation contribuant au développement durable d'un monde plus équitable au cours du $21^{\mathrm{e}}$ siècle? Questions d'orientation, 76, (4), 11-32.

Guichard, J. \& Vignoli, E. (Éd.). (2010). S'orienter: construire sa vie. L'orientation scolaire et professionnelle, 39, 3-182.

Hagen, P. \& Jordan, P. (2008). Theoretical foundations of academic advising. In V. N. Gordon, W. R. Habley \& T. J. Grites (Éd.), Academic advising: A comprehensive handbook (2e éd.) (pp.17-35). San Francisco, CA: Jossey-Bass.

Hirschi, A. (2012). The career resources model: An integrative framework for career counsellors. British journal of guidance \& counselling, 40, (4), 369-383.

Jacques, F. (1982). Différence et subjectivité. Paris: Aubier.

Jacquin, P., \& Juhel, J. (2013). Développement de carrière et construction de sa vie: une nouvelle méthode pour l'orientation tout au long de la vie. L'orientation scolaire et professionnelle, 42, (2), 221-243.

Kraus, K. (2013). Essai sur la relation entre formation continue et conseil - différences et convergences. Éducation permanente suisse, 3, 6-7.

Lakoff, G. \& Johnson M. (1980). Metaphors we live by. Chicago, IL: University of Chicago Press.

Maulini, O. \& Montandon C. (Éd.). (2005). Les formes de l'éducation: variété et variations. Bruxelles: De Boeck.

Mayen, P. (2007). Quelques repères pour analyser les situations dans lesquelles le travail consiste à agir pour et avec un autre. Recherches en éducation, 4, 51-64.

Mayen, P. (Éd.). (2015). Le Conseil en évolution professionnelle. L'activité des bénéficiaires et le métier des conseillers: deux ans d'expérience en Bourgogne. Dijon: Raison et Passions.

Paul, M. (2009). Autour du mot accompagnement. Recherche et formation, 62, 129-139.

Peirce, C. S. $(1878,1879)$. La logique de la science. Deux articles publiés dans la Revue philosophique de la France et de l'étranger "Comment se fixe la croyance» $3 \mathrm{e}$ année, tome VI, décembre 1878, pages 553-569 et "Comment rendre nos idées claires» 4e année, tome VII, janvier 1879, pages 39-57. Paris : Presses Universitaires de France.

Peirce, C.S. (1994). The collected paper of Charles Sanders Peirce (Volumes I-VIII). Charlottesville, VA: Intelex.

Roquet, P. (2009). L'émergence de l'accompagnement. Recherche et formation, 62, 13-24.

Rosenthal, V. (2012). La voix de l'intérieur. Intellectica, 58, 53-90. 
Salini, D. (2013). Inattendus et transformations de signification dans les situations d'information-conseil pour la validation des acquis de l'expérience. Thèse pour le doctorat en sciences de l'éducation, Université de Genève.

Salini, D. (2015). Le conseil en évolution professionnelle et les dynamiques d'anticipation. In P. Mayen (Éd.), Le Conseil en évolution professionnelle. L'activité des bénéficiaires et le métier des conseillers: deux ans d'expérience en Bourgogne (pp. 242-268). Dijon: Raison et Passions.

Sartre, J.-P. (1943). L'être et le néant. Paris: Gallimard.

Savickas, M. L., Nota, L., Rossier, J., Dauwalder, J. P., Duarte, M. E., Guichard, J., Soresi, S., Van Esbroeck, R. \& van Vianen, A. E. M. (2010). Construire sa vie: un paradigme pour l'orientation au 21e siècle. L'orientation scolaire et professionnelle, 39, (1), 5-39.

Schiersmann, C., Ertelt, B. J., Katsarov, J., Mulvey, R., Reid, H. \& Weber, P. (Éd.). (2012). NICE Handbook for the academic training of career guidance and counselling professionals. Heidelberg:

Networkfor Innovation in Career Guidance and Counselling in Europe (NICE). Retrieved from http://www.nice-network.eu/wp-content/uploads/2015/11/NICE_Handbook_ full_version_online.pdf

Theureau, J. (2006). Le cours d'action: méthode développée. Toulouse: Octarès.

Theureau, J. (2015). L'enaction et l'expérience. Toulouse: Octarès.

Varela, F. J., Thompson, E. \& Rosch, E. (1993). L'inscription corporelle de l'esprit. (V. Havelange, trad.). Paris: Seuil. (Original publié en 1991)

Zannini, L. (2005). La tutorship nella formazione degli adulti. Uno sguardo pedagogico. Milano: Guerini.

Mots-clés: Conseil, anticipation, éducation tout au long de la vie, sémiotique, apprentissage informel

\section{Die formative Dimension neuer Ansätze der Erwachsenen- beratung: zwei Fallstudien}

\section{Zusammenfassung}

Ziel dieses Beitrags ist es, die formative Dimension der Erwachsenenberatung anhand der transversalen Analyse von zwei Fallstudien aus der aktuellen Praxis in diesem Bereich hervorzuheben. Die Analyse zeigt auf, welche Beratungssituationen den betroffenen Individuen zu neuen Erkenntnissen verholfen haben. Es handelt sich um folgende Elemente: i) vereinfachter Zugang zu und sachbezogener Umgang mit ausgewählten Informationen, ii) Begleitung bei der Erarbeitung einer Fragestellung im Zusammenhang mit der beruflichen Weiterentwicklung oder eines Bildungsvorhabens. Ermöglicht wird dies durch das von den Beratenden eingesetzte Verfahren, indem zum Transfer von neuen Erkenntnissen die mimetischen, dialogischen und fiktionalen Aspekte besonders präsent sind.

Schlagworte: Beratung, Antizipation, Lebenslanges Lernen, Semiotik, informelles Lernen 


\section{Le dimensioni formative di nuove pratiche di consulenza ad adulti: due studi di caso}

\section{Riassunto}

Questo contributo si focalizza sulle dimensioni formative della consulenza rivolta ad adulti sulla base dell'analisi trasversale di due studi su pratiche recenti in quest'ambito. L'analisi mostra alcuni elementi delle situazioni di consulenza che contribuiscono all'emergere di nuovi apprendimenti presso gli individui coinvolti. Questi elementi sono: i) l'accesso facilitato e la selezione pertinente delle informazioni da proporre, ii) l'accompagnamento all'elaborazione dei loro interrogativi, in riferimento ad un progetto di sviluppo professionale o formativo. Tale accompagnamento è caratterizzato dalla messa in opera di un insieme di modalità di trasmissione di nuove conoscenze, in cui gli aspetti mimetici, dialogici e narrativi sono particolarmente presenti ed efficaci.

Parole chiave: Consulenza, anticipazione, formazione lungo tutto l'arco della vita, semiotica, apprendimento informale

\section{Formative dimensions of news practices of adults counselling: two case studies}

\section{Abstract}

The purpose of this contribution is to highlight the formative implications of adult counselling from the cross-sectional analysis of two case studies on recent practices in the field. This analysis will highlight some elements of counselling situations that contribute to the emergence of new knowledge in the concerned individuals. These elements are: i) facilitated access to a selection of information and its appropriate use; ii) support in the development of their inquiry process, in relation to a professional development or training project. This is made possible through the implementation by counsellors of a set of modalities for the transmission of new knowledge, in which the mimetic, dialogical and fictional dimensions are particularly present.

Keywords: Counselling, anticipation, lifelong learning, semiotic, informal learning 
